\title{
Dynamics of Light Emission in CdMnS Nanoparticles
}

\author{
M. Godlewski ${ }^{a, b, *}$, S. Yatsunenko ${ }^{a}$, \\ K. Drozdowicz-Tomsia ${ }^{c}$, E.M. Goldys ${ }^{c}$, M.R. Phillips $^{d}$, \\ P.J. KLAR ${ }^{e}$ AND W. HeIMBRODT ${ }^{e}$ \\ ${ }^{a}$ Institute of Physics, Polish Academy of Sciences \\ al. Lotników 32/46, 02-668 Warsaw, Poland \\ ${ }^{b}$ Dept. of Mathematics and Natural Sciences College of Science \\ Cardinal S. Wyszyński University, Warsaw, Poland \\ ${ }^{c}$ Div. of Information and Communication Sciences \\ Macquarie University, Sydney, Australia \\ ${ }^{d}$ Microstructural Analysis Unit, UTS, Sydney, Australia \\ ${ }^{e}$ Dept. of Physics and Materials Sciences Center \\ Philipps-University of Marburg, Renthof 5, 35032 Marburg, Germany \\ We demonstrate coexistence of slow and fast components of photolumi- \\ nescence decay of the $\mathrm{Mn}^{2+}$ intra-shell emission in nanoparticles of CdMnS. \\ We explain the observed decrease in PL lifetime of the $\mathrm{Mn}^{2+}$ intra-shell tran- \\ sition by high efficiency of spin dependent magnetic interactions between \\ localized spins of $\mathrm{Mn}^{2+}$ ions and free carriers. This mechanism is enhanced \\ in nanostructures, but it is also present in bulk samples.
}

PACS numbers: 81.07.Wx, 78.55.Et, 78.47.+p, 71.55.Gs, 76.30.Fc, 76.70. $\mathrm{Hb}$

\section{Introduction}

In recent years $\mathrm{Mn}^{2+}$ or rare earth doped II-VI nanoparticles were considered as novel fluorescent labels (for fluorescence labelling) for applications in medicine and biology. Among them CdS, ZnS, CdSe, and $\mathrm{ZnO}$ nanoparticles are the most perspective and thus the most studied.

*corresponding author; e-mail: godlew@ifpan.edu.pl 
In this work we evaluate the influence of confinement effects on light emission characteristics of Mn doped CdS nanoparticles with Mn fractions from 1\% to $30 \%$. Their light emission is studied using a confocal microscopy with a time resolution option. In all samples we observed the coexistence of band gap emission (at room temperature this emission is due to free carriers recombination) and the $\mathrm{Mn}^{2+}$ ( ${ }^{4} T_{1}$ to ${ }^{6} A_{1}$ ) intra-shell emission. In the consequence, we could monitor influence of Mn doping on a rate of the band gap emission. We could also evaluate the influence of $\mathrm{Mn}$-free carrier interactions on a rate of the $\mathrm{Mn}^{2+}$ intra-shell emission, by studying dynamics of these two photoluminescence (PL) bands. These investigations enable us to confirm an enhancement of the $\mathrm{Mn}^{2+}$ intra-shell emission in nanoparticles. Such enhancement was reported for the first time by Bhargava and Gallagher for ZnMnS nanoparticles [1] and then was observed by us for several other systems [2-4].

We evaluate the efficiency of intra-nanoparticles energy migration from the measurements of PL decay kinetics and from the maps of in-plane variations of the PL decay times measured in micro-PL set up. These maps were measured for nanoparticles diluted in a glycol.

\section{Basic concepts}

The ${ }^{4} T_{1}$ to ${ }^{6} A_{1}$ transition of $\mathrm{Mn}^{2+}$ ion is a parity and spin forbidden process. In the consequence, the relevant PL decay times are long (from $\mu \mathrm{s}$ to ms [5]). In particular, this PL decay is long in compounds with weak spin-orbit interaction, such as $\mathrm{CdS}$ or ZnS (ms decay). Surprisingly, Bhargava and Gallagher reported a dramatic shortening of the $\mathrm{Mn}^{2+} \mathrm{PL}$ decay time (by six orders in magnitude) in nanoparticles of ZnMnS [1]. Such shortening of the PL decay time could not be related to competition of some nonradiative recombination processes, since shortening of the PL decay time was observed together with a high quantum efficiency of the intra-shell PL. The effect was tentatively explained by a model of a quantum confined atom. In this model, quantum confinement affects $\mathrm{Mn}^{2+}$ $3 d$-type wave function ( $s p-d$ hybridization with host states) and thus relaxes parity and spin selection rules for the $\mathrm{Mn}^{2+}$ intra-shell transition [1].

In the following experimental works the model was either confirmed or rejected [6-9], resulting in a very confusing situation. For example, Bol and Meijerink claimed that the fast PL decay is an artefact, and that it must be due to another PL, which overlaps with the $\mathrm{Mn}^{2+}$ intra-shell PL [6]. "Normal" ms decay was observed for $\mathrm{Mn}^{2+}$ intra-shell PL in nanoparticles of ZnMnS [6] and also CdMnS [7], which was in disagreement with the model of Bhargava. In turn, the authors of [8] and [9] assumed that the observation of Bhargava is correct, but they proposed another theoretical model accounting for the observed PL lifetime shortening.

In this work we confirm experimentally the observation of Bhargava and Gallagher, demonstrating the appearance of a short component of the $\mathrm{Mn}^{2+} \mathrm{PL}$ decay 
in nanoparticles of CdMnS. We propose however another mechanism responsible for this fast PL decay.

\section{Experimental results and discussion}

The experiments were performed for nanostructures of CdMnS, synthesized in the mesoporous MCM-41 $\mathrm{SiO}_{2}$ matrices [10], with grains of 5 to $40 \mathrm{~nm}$ size, as estimated in the scanning electron microscopy (SEM) investigations (see Fig. 1). PL, time-resolved PL, electron spin resonance (ESR), SEM, and cathodoluminescence (CL) experiments were performed. The set ups used in the present study are described elsewhere [2-4].

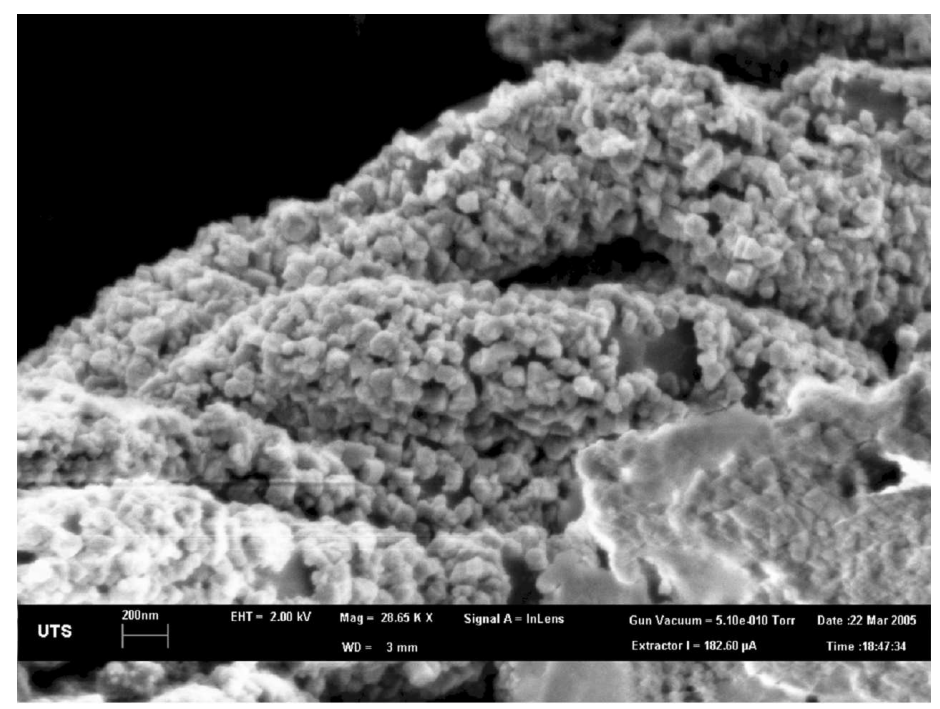

Fig. 1. High resolution SEM image of CdMnS nanoparticles (with 1\% Mn fraction) measured at $2 \mathrm{kV}$ accelerating voltage and at the room temperature.

Some groups claimed that transition metal and rare earth (RE) ions do not enter nanoparticles of II-VI compounds (see the recent review by $\mathrm{Hu}$ and Zhang and references therein [11]). We thus first checked whether $\mathrm{Mn}^{2+}$ ions are present in the nanoparticles studied by us by performing ESR experiments. Strong magnetic resonance of $\mathrm{Mn}^{2+}$ ions was observed with a well-resolved six-line hyperfine (HF) structure, as shown in Fig. 2 for CdMnS with $20 \%$ Mn fraction. The HF structure was observed for all nanoparticles studied by us. This was a very surprising result, since the HF structure is typically not resolved in bulk II-Mn-VI samples for increased Mn fractions, typically above $0.5 \%$ of $\mathrm{Mn}$.

From microwave power dependence of the ESR spectrum we found that this spectrum is due to an overlap of two $\mathrm{Mn}^{2+}$ - related signals - the first ESR signal shows the characteristic 6 lines HF structure, the second ESR signal (a broad, 


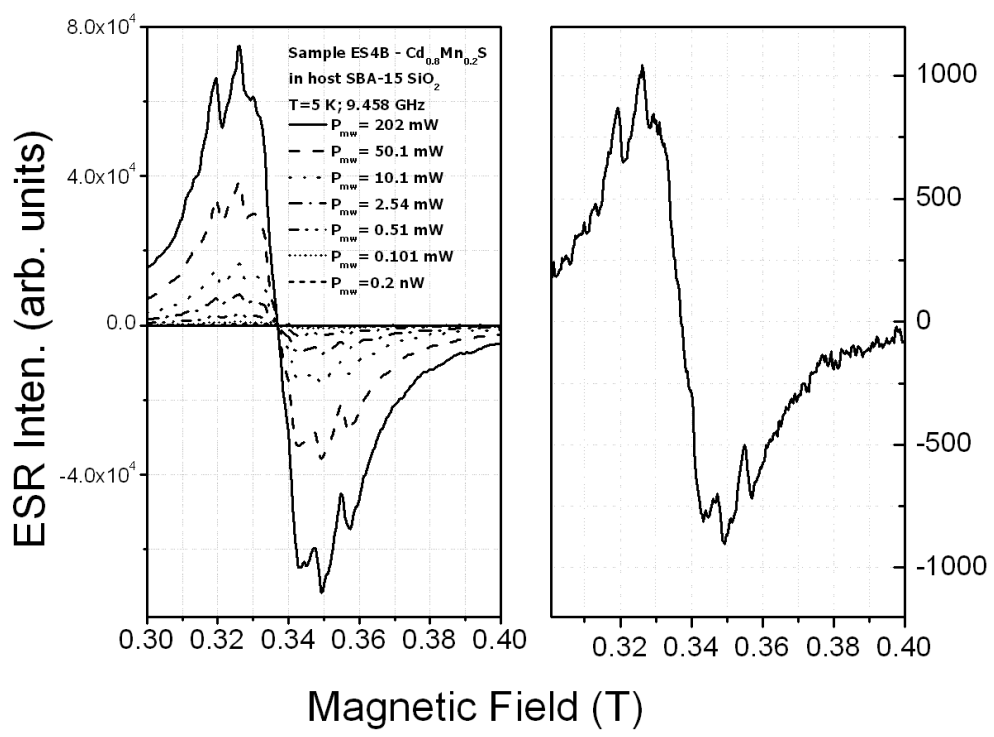

Fig. 2. ESR spectrum (right, for $1 \mathrm{~mW}$ ) and its microwave power dependence (left) for CdMnS nanoparticles with $20 \% \mathrm{Mn}$ fraction measured at liquid helium temperature.

featureless line) has the same $g$-factor as the first one, it is why we also attribute this signal to the magnetic resonance of $\mathrm{Mn}^{2+}$ ions. We observed similar ESR spectra for ZnMnS nanoparticles [4] and we related them to the ESR signals of isolated $\mathrm{Mn}^{2+}$ ions at surfaces of nanoparticles (the one with the HF structure), and to the ESR signal (the broad one) coming from the volume of nanoparticles.

The first ESR signal (better seen in Fig. 2 (right)) saturates faster with an increasing microwave power. The second ESR signal (the underlying broad one) becomes dominant with an increasing microwave power and saturates slower than the first one, indicating a significant shortening of a spin relaxation time for $\mathrm{Mn}^{2+}$ ions in a volume of nanoparticles. This we explain by efficient $\mathrm{Mn}-\mathrm{Mn}$ spin cross-relaxation.

The PL (CL) spectrum of CdMnS nanoparticles consists of three PL (CL) bands. The first one is the band edge emission, which is resolved even at the room temperature (Fig. 3). In CL the band edge emission was observed only at large excitation density. The second emission, dominating at low and moderate excitation conditions (see Fig. 3), is the $\mathrm{Mn}^{2+}$ intra-shell PL. This emission is accompanied by another one (the third PL) at low energy wing. The origin of this low energy PL band, extending down to $1.6 \mathrm{eV}$, remains unknown. It can be due to some underlying PL of a different origin, or is due to $\mathrm{PL}$ emission of $\mathrm{Mn}^{2+}$ ions in a different environment. The latter can be due to different spectral responses of isolated $\mathrm{Mn}^{2+}$ ions and $\mathrm{Mn}^{2+}$ associates, complexes, etc. We however reject such explanation of this PL band, based on the results of CL investigations, described below. 


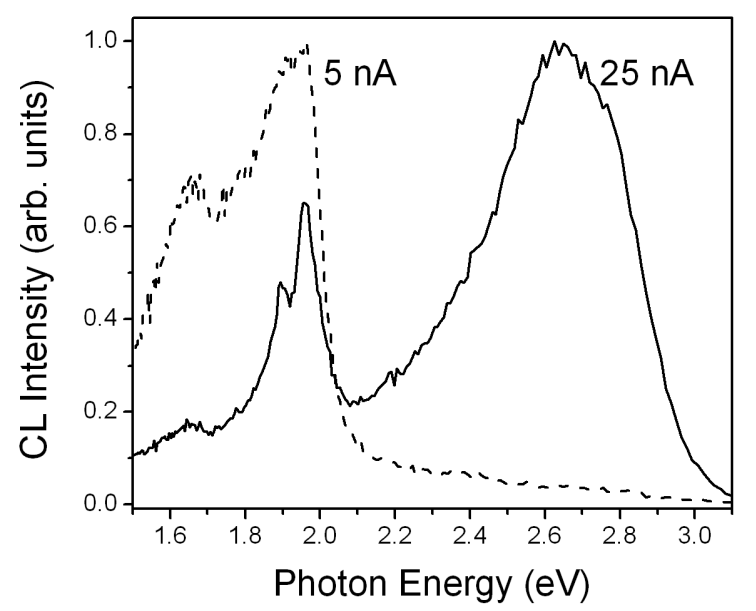

Fig. 3. Room temperature cathodoluminescence spectra of CdMnS nanoparticles (with $1 \% \mathrm{Mn}$ fraction), measured at 5 and $25 \mathrm{nA}$ excitation density. Spectra were normalized to a constant intensity, to depict a better evolution of their spectral shape.

In the CL (see Fig. 3) we noticed that the CL emission changes its character at "high" density of the e-beam excitation. By "high" current density we mean here e-beam current of about 25 nanoampers. Intensity of the $\mathrm{Mn}^{2+}$ intra-shell emission increases first with an increasing excitation density. However, at high density of excitation (e-beam currents above $10 \mathrm{nA}$ ) charging effects become important. Then, the CL spectrum changes and the change is not reversible. The $\mathrm{Mn}^{2+}$ intra-shell emission disappears and is replaced by a new structured emission peaking at $1.95 \mathrm{eV}$, as shown in Fig. 3. We explain this change by oxidization effects, i.e., we propose that $\mathrm{Mn}$ ions are oxidized forming some $\mathrm{Mn}_{x} \mathrm{O}_{y}$ oxides. Whereas the $\mathrm{Mn}^{2+}$ intra-shell emission is removed, the low energy wing emission is not affected by charging effects at high excitation density. We thus claim that this emission must be of a different origin and we propose that this emission is defect-related.

PL decay kinetics was measured by setting detection at the PL maxima of the $\mathrm{Mn}^{2+}$ intra-shell emission (Fig. 4) and the band edge emission (not shown in Fig. 4). For these investigations we used a micro-PL set up with a time resolution. Pulsed $405 \mathrm{~nm}$ laser diode was used for the PL excitation with $40 \mathrm{MHz}$ repetition rate, i.e., the above band gap excitation was used. At such excitation conditions all slow components of the PL decay were saturated and only the fast ones could be studied, with the $100 \mathrm{ps}$ time resolution.

Figure 4 shows the rise and decay in the $\mathrm{Mn}^{2+} \mathrm{PL}$ in the first $5 \mathrm{~ns}$ after turning on and off the excitation. The $\mathrm{Mn}^{2+}$ intra-shell emission shows a very pronounced fast component of the PL decay, which is observed together with slow decay components, not resolved in the experiment shown in Fig. 4. We could partly resolve the slow PL decay components by reducing laser repetition rate down to 


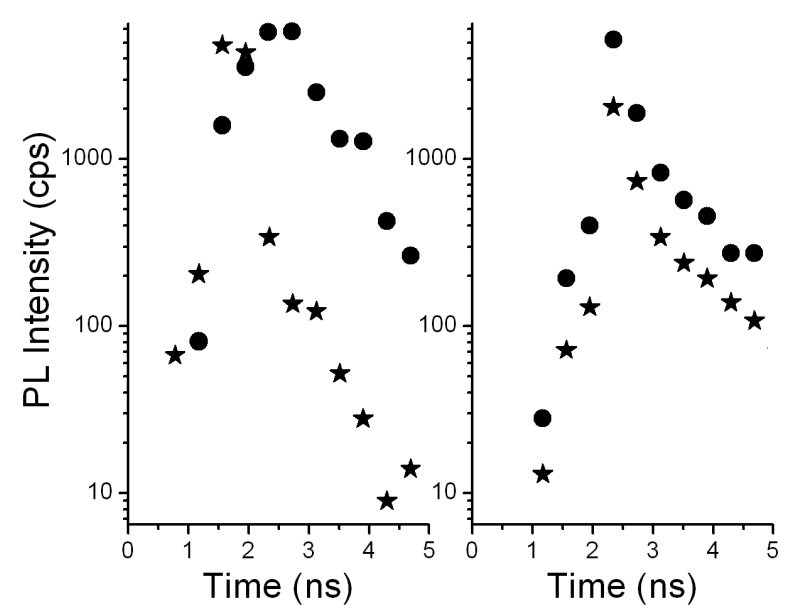

Fig. 4. PL kinetics of $\mathrm{Mn}^{2+}$ intra-shell emission in CdMnS (Mn fraction of $1 \%$ (left) and $30 \%$ (right)) measured at pulsed $405 \mathrm{~nm}$ excitation, at the room temperature, and at $1 \%(\bullet)$ and $100 \%$ (stars) excitation power.

$5 \mathrm{MHz}$, and we could evaluate them by studying PL kinetics in a "conventional" PL decay set up with ms time resolution. From these investigations we concluded that fast and slow PL decay components coexist.

For the further discussion it is important to notice that the fast component of the $\mathrm{Mn}^{2+} \mathrm{PL}$ decay is analogous to the fast component of the PL decay of the band edge emission. Moreover, we noticed that the fast PL decay component critically depends on excitation density (see Fig. 4), and is also affected by $\mathrm{Mn}$ fraction in nanoparticles. To evidence these dependences we compare in Fig. 4 the PL kinetics observed for low (1\%) and high (100\%) excitation densities, and for two types of nanoparticles - the one with small and the one with large Mn fractions. We underline (see Fig. 4) that sub-ns component of the PL decay (200 ps to $600 \mathrm{ps}$ ) is very much enhanced once the concentration of excited free carriers is increased. This is why we do not give exact values of this PL decay component, but their range, since the lifetime measured depended on the excitation conditions. The effect is more pronounced in nanoparticles with the lower Mn fraction (Fig. 4 left).

Maps of the in-plane variations of the PL decay times were measured for the $\mathrm{Mn}^{2+}$ PL and the band edge PL. In this study we measured PL decay at different areas of accumulated nanoparticles and compared decay kinetics observed for the $\mathrm{Mn}^{2+} \mathrm{PL}$ and the band edge PL. Based on the comparison of these PL decays and their in-plane variations we propose that the fast component (hundreds of picoseconds) observed in the $\mathrm{Mn}^{2+} \mathrm{PL}$ decay is a measure of a lifetime of free carriers in our nanoparticles. Once free carriers are trapped, or once they recombine, the rate of the $\mathrm{Mn}^{2+} \mathrm{PL}$ decay is reduced. This explains the observed drop in the PL intensity of $\mathrm{Mn}^{2+}$ intra-shell emission with a characteristic lifetime 
of free carriers. We have drawn a similar conclusion from the recent investigations of $\mathrm{ZnMnS}$ nanoparticles and CdMnTe quantum dots [2-4]. Co-excitation of free carriers and excited $\mathrm{Mn}^{2+}$ ions is necessary for observation of shortening of the $\mathrm{Mn}^{2+} \mathrm{PL}$ decay time $[2-4]$.

We further analyzed the role of $\mathrm{Mn}-\mathrm{Mn}$ spin flip interactions (cross-relaxation). Such interactions can also reduce PL decay time of the $\mathrm{Mn}^{2+}$ intra-shell emission, by relaxing spin selection rules [2-4]. Based on the present PL kinetics investigations we confirm that these interactions are in fact important. We observed shortening of the $\mathrm{Mn}^{2+} \mathrm{PL}$ decay in nanoparticles with larger $\mathrm{Mn}$ fractions (see Fig. 4, right). This shortening can be due to an increased role of the $\mathrm{Mn}-\mathrm{Mn}$ cross-relaxation, but can also reflect an increased role of $\mathrm{Mn}$ to $\mathrm{Mn}$ energy transfer processes.

Both these interactions cannot alone account for the fast component of the $\mathrm{PL}$ decay, the one observed by Bhargava for $\mathrm{ZnMnS}$ nanoparticles and also by us for CdMnS nanoparticles. Our theoretical estimations suggest that the Mn-Mn cross-relaxation can shorten the $\mathrm{Mn}^{2+} \mathrm{PL}$ decay time by at most four orders in magnitude, and not by six. The Mn-free carriers spin-flip interactions must thus be dominant.

\section{Conclusions}

Concluding, we demonstrate that the fast component of the $\mathrm{Mn}^{2+} \mathrm{PL}$ decay is present in nanoparticles of CdMnS. This effect is however not related to quantum confinement of $3 d$ wave function of $\mathrm{Mn}^{2+}$ ions, as proposed by Bhargava and Gallagher, but is due to efficient Mn-free carriers spin flip interactions. These interactions are enhanced in low dimensional structures. We also expect that the similar mechanism is active in RE doped nanostructures, explaining the origin of shortening of the PL decay times often observed in such systems. This observation suggests that both $\mathrm{Mn}$ and RE doped nanoparticles are attractive candidates for fluorescence labels (efficient emission, high recombination rate).

\section{Acknowledgments}

This work was partly supported by the grant number PBZ-KBN-044/P03/2001 of the State Committee for Scientific Research (Poland).

\section{References}

[1] R.N. Bhargava, D. Gallagher, Phys. Rev. Lett. 72, 416 (1994).

[2] M. Godlewski, V.Yu. Ivanov, A. Khachapuridze, S. Yatsunenko, Physica Status Solidi B 229, 533 (2002). 
[3] M. Godlewski, S. Yatsunenko, A. Khachapuridze, V.Yu. Ivanov, Z. Gołacki, G. Karczewski, P.J. Bergman, P.J. Klar, W. Heimbrodt, M.R. Phillips, J. Alloys Compounds 380, 45 (2004).

[4] M. Godlewski, S. Yatsunenko, V.Yu. Ivanov, A. Khachapuridze, K. Świạtek, E.M. Goldys, M.R. Phillips, P.J. Klar, W. Heimbrodt, Acta Phys. Pol. A 107, 65 (2005).

[5] W. Busse, H.-E. Gumlich, B. Meissner, D. Theis, J. Lumin. 12/13, 693 (1976).

[6] A.A. Bol, A. Meijerink, Phys. Rev. B 58, R15997 (1998).

[7] M.V. Artemyev, L.I. Gurinovich, A.P. Stupak, S.V. Gaponenko, Phys. Status Solidi B 224, 191 (2001).

[8] Kuo Yan, Chnagkui Duan, Yi Ma, Shanda Xia, J.-C. Krupa, Phys. Rev. B 58, 13585 (1998).

[9] Nguyen Que Huong, J.L. Birman, Phys. Rev. B 69, 085321 (2004).

[10] L. Chen, P.J. Klar, W. Heimbrodt, F. Brieler, M. Fröba, Appl. Phys. Lett. 76, 3531 (2000).

[11] H. Hu, W. Zhang, to be published. 